\title{
A Lagrangian effective field theory
}

\author{
Zvonimir Vlah ${ }^{a, b}$ Martin White ${ }^{c, d}$ Alejandro Aviles $^{d}$ \\ ${ }^{a}$ Stanford Institute for Theoretical Physics and Department of Physics, Stanford University, \\ Stanford, CA 94306, USA \\ ${ }^{b}$ Kavli Institute for Particle Astrophysics and Cosmology, SLAC and Stanford University, \\ Menlo Park, CA 94025, USA \\ ${ }^{c}$ Department of Physics, University of California, Berkeley, CA 94720 \\ ${ }^{d}$ Department of Astronomy, University of California, Berkeley, CA 94720 \\ E-mail: zvlah@stanford.edu, mwhite@berkeley.edu, aviles@berkeley.edu
}

\begin{abstract}
We have continued the development of Lagrangian, cosmological perturbation theory for the low-order correlators of the matter density field. We provide a new route to understanding how the effective field theory (EFT) of large-scale structure can be formulated in the Lagrandian framework and a new resummation scheme, comparing our results to earlier work and to a series of high-resolution N-body simulations in both Fourier and configuration space. The 'new' terms arising from EFT serve to tame the dependence of perturbation theory on small-scale physics and improve agreement with simulations (though with an additional free parameter). We find that all of our models fare well on scales larger than about two to three times the non-linear scale, but fail as the non-linear scale is approached. This is slightly less reach than has been seen previously. At low redshift the Lagrangian model fares as well as EFT in its Eulerian formulation, but at higher $z$ the Eulerian EFT fits the data to smaller scales than resummed, Lagrangian EFT. All the perturbative models fare better than linear theory.
\end{abstract}

Keywords: cosmological parameters from LSS - power spectrum - baryon acoustic oscillations - galaxy clustering

ArXiv ePrint: YYMM.NNNNN 


\section{Contents}

1 Introduction 1

2 Background $\quad 2$

3 Effective equations of motion $\quad 3$

4 Cumulants 5

$\begin{array}{llr}5 & \text { Resummation schemes } & 8\end{array}$

6 Comparison to Eulerian theory $\quad 9$

$\begin{array}{lll}7 & \text { Zeldovich EFT } & 10\end{array}$

8 Comparison to simulations $\quad 10$

9 Conclusions 13

$\begin{array}{ll}\text { A Useful identities } & 15\end{array}$

B IR resummation $\quad 16$

\section{Introduction}

The Universe we observe contains structure on essentially all scales, which is believed to arise from a process of gravitational instability acting on small perturbations laid down in the very early Universe. The theory of the evolution of these perturbations in their linear phase is now well developed, and the exceptional agreement between theory and observation for the anisotropies in the cosmic microwave background is one of the triumphs of modern cosmology [1]. Studying these evolution of these perturbations in the modern Universe, when linear perturbation theory is breaking down, is more difficult but can be a powerful probe of cosmology $[2,3]$.

Traditionally perturbation theories in cosmology have been formulated primarily in 'Eulerian form', wherein the matter is treated as a pressureless fluid and a perturbative solution to the continuity, Euler and Poisson equations is obtained (see e.g. ref [4] for a review). Zeldovich [5] proposed a 'Lagrangian form' of perturbation theory, in which one solves perturbatively for the displacement field between the initial and final positions of fluid elements (or dark matter particles). Lagrangian theories tend to fare much better at describing the large-scale advection of matter, and allow a simpler route to understanding redshift-space distortions and the clustering of biased objects [6, 7]. Lagrangian perturbation theory (LPT) has now been well developed in the literature [6-19] and it has been applied to understanding the broadening and shifts of the baryon acoustic oscillation (BAO) peak [20-22], how reconstruction removes these effects [23-26], to study higher order statistics [27, 28] and as the base for a new version of the halo model [29].

One of the drawbacks of both the Eulerian and Lagrangian schemes in their classical formulation is that they are only valid prior to shell crossing and that they treat non-linear 
scales as if they were perturbative. One method for treating these deficiencies is through effective field theory techniques, in which 'effective' equations of motion which depend only on smoothed fields are solved [30-35]. A description of a Lagrangian effective theory was presented in [36], and used in [37] as a technique for doing IR resummation. An alternative formulation specialized to the case of one spatial dimension was presented in [38].

In this paper we present a new derivation of the Lagrangian effective field theory, show how it can be coupled with existing resummation schemes and compare our results to a series of N-body simulations in both Fourier and configuration space. The outline of the paper is as follows. In Section 2 we present some background on LPT and effective field theory, introducing our notation and conventions. Section 3 shows how one can derive an effective equation of motion for the Lagrangian displacement which can be iteratively solved and which contains the effects of short-wavelength perturbations as a series of corrections to the usual Lagrangian perturbation theory expansion. The cumulants of the Lagrangian displacement are all that are needed to derive the correlation function and power spectrum of the mass, and in section 4 we discuss the key cumulants and the counter terms which are introduced by EFT. Section 5 introduces our main results, a resummed version of 1-loop Lagrangian perturbation theory which incorporates the EFT corrections to lowest order. This is contrasted with the Eulerian formulation in section 6 and to compared to N-body simulations in section 8 . We summarize our major findings in section 9, while some technical details are relegated to appendices.

\section{Background}

We shall be primarily interested in the 2-point statistics of the fractional density perturbation, $\delta=\rho / \bar{\rho}-1$, with the correlation function defined as $\xi(\mathbf{r})=\langle\delta(\mathbf{x}) \delta(\mathbf{x}+\mathbf{r})\rangle$, and its Fourier transform, the power spectrum $P(\mathbf{k})$, defined as $\left\langle\delta(\mathbf{k}) \delta\left(\mathbf{k}^{\prime}\right)\right\rangle=(2 \pi)^{3} \delta_{D}\left(\mathbf{k}+\mathbf{k}^{\prime}\right) P(\mathbf{k})$ where angled brackets signify an ensemble average. Here and throughout $\delta_{D}$ denotes the 3-dimensional Dirac delta function, and we use the Fourier transform convention

$$
F(\mathbf{x})=\int \frac{d^{3} k}{(2 \pi)^{3}} F(\mathbf{k}) e^{i \mathbf{k} \cdot \mathbf{x}} .
$$

The Lagrangian approach to cosmological structure formation was developed in [5-9, 11, $13,15,19]$ and traces the trajectory of an individual fluid element through space and time. For a fluid element located at position $\mathbf{q}$ at some initial time $t_{0}$, its position at subsequent times can be written in terms of the Lagrangian displacement field $\boldsymbol{\Psi}$,

$$
\mathbf{x}(\mathbf{q}, t)=\mathbf{q}+\mathbf{\Psi}(\mathbf{q}, t),
$$

where $\mathbf{\Psi}\left(\mathbf{q}, t_{0}\right)=0$. Every element of the fluid is uniquely labeled by $\mathbf{q}$ and $\mathbf{\Psi}(\mathbf{q}, t)$ fully specifies the evolution. Once $\mathbf{\Psi}(\mathbf{q})$ is known, the density field at any time is simply

$$
1+\delta(\mathbf{x})=\int d^{3} q \delta_{D}[\mathbf{x}-\mathbf{q}-\mathbf{\Psi}(\mathbf{q})] \quad \Rightarrow \quad \delta(\mathbf{k})=\int d^{3} q e^{i \mathbf{k} \cdot \mathbf{q}}\left(e^{i \mathbf{k} \cdot \mathbf{\Psi}(\mathbf{q})}-1\right)
$$

The evolution of $\boldsymbol{\Psi}$ is governed by $\partial_{t}^{2} \mathbf{\Psi}+2 H \partial_{t} \boldsymbol{\Psi}=-\nabla \Phi(\mathbf{q}+\boldsymbol{\Psi})$. We shall work throughout in terms of conformal time, $d \eta=d t / a$, and write $\mathcal{H}=a H$ for the conformal Hubble parameter. The equation of motion is thus

$$
\ddot{\mathbf{\Psi}}+\mathcal{H} \dot{\boldsymbol{\Psi}}=-\nabla \Phi(\mathbf{q}+\mathbf{\Psi})
$$


where overdots indicate derivatives w.r.t. conformal time. In section 3 we describe how we account for small-scale structures in eq. 2.4 such that the remaining fields contain only small, long-wavelength perturbations which are amenable to treatment via LPT. In LPT one finds a perturbative solution for $\boldsymbol{\Psi}$ :

$$
\boldsymbol{\Psi}(\mathbf{q}, t)=\boldsymbol{\Psi}^{(1)}(\mathbf{q}, t)+\mathbf{\Psi}^{(2)}(\mathbf{q}, t)+\mathbf{\Psi}^{(3)}(\mathbf{q}, t)+\cdots
$$

with the first order solution, linear in the density field, being the Zel'dovich approximation [5]. Higher order solutions are specified in terms of integrals of higher powers of the linear density field $[18,19]$ (see eq. 3.7). To these perturbative terms are then added a series of 'extra' terms, which encapsulate the effect of the small-scale physics which is missing in the perturbative treatment.

\section{Effective equations of motion}

The dynamics of our system are specified by the equations of motion eq. (2.4) for which we shall attempt a perturbative solution. However on small scales the fluctuations are large and not amenable to a perturbative treatment, which has led the community to investigate effective field theory descriptions which can provide an accurate description of the longwavelength physics without detailed knowledge of the short-wavelength dynamics. Following the philosophy of effective field theory as it is normally used in the cosmology community [3035] we shall smooth eq. (2.4) to remove small scales, accounting for the small-scale physics with a series of counter terms each containing constants we are not be able to determine from the theory itself. As emphasized by [35] this method has the drawback of removing too many small-scale terms, including those generated by two long wavelength modes, however for the low orders of interest to us it is sufficient. We shall also restrict ourselves to the longitudinal degrees of freedom, since again at the orders we work the effects of vorticity can be safely ignored. In this section we have tried to make explicit connection with the earlier work of ref. [36], who presented an investigation of Lagrangian EFT, sometimes adopting their notation to make the connections most clear.

We smooth eq. (2.4) in q-space using a filter $W_{R}\left(\mathbf{q}, \mathbf{q}^{\prime}\right)$, splitting the system into $L$-long and $S$-short wavelength modes, e.g.

$$
\boldsymbol{\Psi}_{L}(\mathbf{q})=\int d^{3} q^{\prime} W_{R}\left(\mathbf{q}, \mathbf{q}^{\prime}\right) \Psi\left(\mathbf{q}^{\prime}\right) \quad, \quad \Psi_{S}\left(\mathbf{q}, \mathbf{q}^{\prime}\right)=\mathbf{\Psi}\left(\mathbf{q}^{\prime}\right)-\Psi_{L}(\mathbf{q})
$$

from which it follows that the integral of $W_{R} \boldsymbol{\Psi}_{S}$ over $\mathbf{q}^{\prime}$ vanishes. By analogy we also define $\delta_{L}$ as the long-wavelength component of the density perturbation (using eq. 2.3 with $\boldsymbol{\Psi}_{L}$ ) and $\Phi_{L}$ as the gravitational potential sourced by $\delta_{L}$

$$
\nabla^{2} \Phi_{L}=\frac{3}{2} \mathcal{H}^{2} \Omega_{m} \delta_{L}
$$

(this is a different definition than ref. [36], who perform an additional expansion for the source of the Poisson equation). The short-wavelength density and potential are then $\Phi_{S}=\Phi-\Phi_{L}$ and $\delta_{S}=\delta-\delta_{L}$ 
Smoothing the equation of motion for $\boldsymbol{\Psi}$

$$
\begin{aligned}
\ddot{\mathbf{\Psi}}_{L}(\mathbf{q})+\mathcal{H} \dot{\mathbf{\Psi}}_{L}(\mathbf{q}) & =-\int d^{3} q^{\prime} W_{R}\left(\mathbf{q}, \mathbf{q}^{\prime}\right) \nabla \Phi\left(\mathbf{q}^{\prime}+\mathbf{\Psi}\left(\mathbf{q}^{\prime}\right)\right) \\
& =-\int d^{3} q^{\prime} W_{R}\left(\mathbf{q}, \mathbf{q}^{\prime}\right) \nabla \Phi_{L}\left(\mathbf{q}^{\prime}+\mathbf{\Psi}\left(\mathbf{q}^{\prime}\right)\right)-\int d^{3} q^{\prime} W_{R}\left(\mathbf{q}, \mathbf{q}^{\prime}\right) \nabla \Phi_{S}\left(\mathbf{q}^{\prime}+\mathbf{\Psi}\left(\mathbf{q}^{\prime}\right)\right) \\
& =-\nabla \Phi_{L}\left(\mathbf{q}+\mathbf{\Psi}_{L}(\mathbf{q})\right)+\mathbf{a}_{S}\left(\mathbf{q}, \mathbf{\Psi}_{L}(\mathbf{q})\right) .
\end{aligned}
$$

Apart from the $\Phi$ dependence on $\boldsymbol{\Psi}_{L}$ we shall not need explicit expressions for the sources in what follows, since their structure will be dictated by symmetry. However to make contact with ref. [36] we note that the second term on the r.h.s. can be written as a multipole expansion having contributions from $\Phi_{L}$ and $\Phi_{S}$. The $\Phi_{L}$ piece is

$$
\begin{aligned}
\mathbf{a}_{S}(\mathbf{q}) & \ni \nabla \Phi_{L}\left(\mathbf{q}+\mathbf{\Psi}_{L}(\mathbf{q})\right)-\int d^{3} q^{\prime} W_{R}\left(\mathbf{q}, \mathbf{q}^{\prime}\right) \nabla \Phi_{L}\left(\mathbf{q}^{\prime}+\mathbf{\Psi}\left(\mathbf{q}^{\prime}\right)\right) \\
& =\int \frac{d^{3} k}{(2 \pi)^{3}}(i \mathbf{k}) \Phi_{L}(\mathbf{k}) e^{i \mathbf{k} \cdot\left(\mathbf{q}+\mathbf{\Psi}_{L}(\mathbf{q})\right)} \int d^{3} q^{\prime}\left(W_{R}\left(\mathbf{q}, \mathbf{q}^{\prime}\right) e^{i \mathbf{k} \cdot \Psi_{S}\left(\mathbf{q}, \mathbf{q}^{\prime}\right)}-\delta_{D}\left(\mathbf{q}-\mathbf{q}^{\prime}\right)\right) e^{i \mathbf{k} \cdot \delta \mathbf{q}} \\
& =\sum_{n=2}^{\infty} \frac{i^{n}}{n !} \int \frac{d^{3} k}{(2 \pi)^{3}}\left(i \mathbf{k} k_{i_{1}} \ldots k_{i_{n}}\right) \Phi_{L}(\mathbf{k}) e^{i \mathbf{k} \cdot\left(\mathbf{q}+\Psi_{L}(\mathbf{q})\right)} \\
& \times \int d^{3} q^{\prime} W_{R}\left(\mathbf{q}, \mathbf{q}^{\prime}\right)\left[\delta \mathbf{q}+\mathbf{\Psi}_{S}\left(\mathbf{q}, \mathbf{q}^{\prime}\right)\right]_{i_{1}} \ldots\left[\delta \mathbf{q}+\mathbf{\Psi}_{S}\left(\mathbf{q}, \mathbf{q}^{\prime}\right)\right]_{i_{n}} \\
& =\sum_{n=2}^{\infty} \frac{i^{n}}{n !} Q_{S}^{i_{1} \ldots i_{n}}(\mathbf{q}) \int \frac{d^{3} k}{(2 \pi)^{3}}\left(i \mathbf{k} k_{i_{1}} \ldots k_{i_{n}}\right) \Phi_{L}(\mathbf{k}) e^{i \mathbf{k} \cdot\left(\mathbf{q}+\mathbf{\Psi}_{L}(\mathbf{q})\right)} \\
& =-\frac{1}{2} Q_{S}^{i j}(\mathbf{q}) \nabla \nabla_{i} \nabla_{j} \Phi_{L}\left(\mathbf{q}+\mathbf{\Psi}_{L}(\mathbf{q})\right)+\ldots
\end{aligned}
$$

where we have defined multipole moments of the short displacement field

$$
Q_{S}^{i_{1} \ldots i_{n}}(\mathbf{q})=\int d^{3} q^{\prime} W_{R}\left(\mathbf{q}, \mathbf{q}^{\prime}\right)\left[\delta \mathbf{q}+\mathbf{\Psi}_{S}\left(\mathbf{q}, \mathbf{q}^{\prime}\right)\right]_{i_{1}} \ldots\left[\delta \mathbf{q}+\mathbf{\Psi}_{S}\left(\mathbf{q}, \mathbf{q}^{\prime}\right)\right]_{i_{n}} .
$$

and we note that the dipole moment is missing since the averages of $\boldsymbol{\Psi}_{S}$ and $\delta \mathbf{q}$ both vanish. The acceleration due to the short wavelength modes follows a similar structure, $\mathbf{a}_{S}(\mathbf{q}) \ni$ $-\nabla \Phi_{S}\left(\mathbf{q}+\mathbf{\Psi}_{L}(\mathbf{q})\right)-\frac{1}{2} Q_{S}^{i j}(\mathbf{q}) \nabla \nabla_{i} \nabla_{j} \Phi_{S}\left(\mathbf{q}+\mathbf{\Psi}_{L}(\mathbf{q})\right)+\ldots$, representing the contribution of $\Phi_{S}$ to the evolution of $\boldsymbol{\Psi}_{L}$. Note that $\Phi_{S}$ depends on the long-wavelength displacement (and hence density) through its argument but there is no contribution to the center of mass so the dependence is through tidal fields of $\Phi_{L}$.

Regardless of the particular form for the expansion we have

$$
\ddot{\mathbf{\Psi}}_{L}+\mathcal{H} \dot{\mathbf{\Psi}}_{L}=-\nabla \Phi_{L}\left(\mathbf{q}+\mathbf{\Psi}_{L}(\mathbf{q})\right)+\mathbf{a}_{S}\left(\mathbf{q}, \mathbf{\Psi}_{L}(\mathbf{q})\right)
$$

where the first term can be treated perturbatively and the "extra" term, $\mathbf{a}_{S}$, contains sources of displacement that arise from small-scale modes which may not be well captured by perturbation theory. ${ }^{1}$ The contribution to $\boldsymbol{\Psi}_{L}$ which is $n^{\text {th }}$ order in the long-wavelength, linear theory perturbation, $\delta_{0}$, is

$$
\mathbf{\Psi}_{L}^{(n)}(\mathbf{k})=\frac{i D^{n}}{n !} \int \frac{d^{3} k_{1}}{(2 \pi)^{3}} \cdots \frac{d^{3} k_{n}}{(2 \pi)^{3}}(2 \pi)^{3} \delta_{D}\left(\sum_{j} \mathbf{k}_{j}-\mathbf{k}\right) \mathbf{L}_{n}\left(\mathbf{k}_{1}, \cdots, \mathbf{k}_{n}\right) \delta_{0}\left(\mathbf{k}_{1}\right) \cdots \delta_{0}\left(\mathbf{k}_{n}\right)
$$

\footnotetext{
${ }^{1}$ In our approach all of the short-distance terms, including the multipole expansion of $\Phi$, are absorbed in $\mathbf{a}_{S}$ in contrast to ref. [36]. Thus the 'additional' terms all arise from $\mathbf{a}_{S}$.
} 
with $D$ the linear growth rate and the $\mathbf{L}_{n}$ given in e.g. $[6,18,19]$ with the lowest order term being simply $\mathbf{L}_{1}(\mathbf{k})=\mathbf{k} / k^{2}$.

The additional contributions to $\boldsymbol{\Psi}_{L}$ come from the source term, $\mathbf{a}_{S}$, which we must integrate against the Green's function. We cannot compute this term from first principles, but we can parameterize its dependence on $\boldsymbol{\Psi}_{L}$ with a small number of terms which are constrained by the symmetries of the problem. The first contribution is a 'stochastic' term, $\mathcal{S}$, which is independent of the long-wavelength modes. The first non-trivial dependence on the long-wavelength density that transforms as a vector must ${ }^{2}$ be proportional to $\nabla \delta_{0}$. Grouping the contributions by their dependence on $\delta_{0}$ and the number of spatial derivatives (see ref. [36, 38, 39] for similar expansions) and keeping only the lowest order terms we thus have

$$
\mathbf{\Psi}_{L} \ni \mathcal{S}+\frac{1}{2} \alpha_{1} \nabla \delta_{0}+\cdots
$$

with $\alpha_{1}$ an undetermined coefficient and $\mathcal{S}$ uncorrelated with $\delta_{0}$. This is the same, lowest order contribution as derived in [36, 38]. As we shall see, these terms lead to corrections to the displacement power spectrum and additionally serve to tame contributions from high $k$ modes in the usual perturbative treatment.

Formally the expansion above is in powers of derivatives or $k / \Lambda$, with $\Lambda$ some cut-off scale chosen to render the perturbation theory integrals well behaved. The theory is $\Lambda$ independent if all terms in the expansion are kept, but doing so introduces an infinite number of undetermined constants. If we truncate the expansion at a fixed order, and if $k<\Lambda$, higher order terms are suppressed by powers of $k / \Lambda<1$ and thus should be numerically smaller than the terms kept (unless some constants artificially make some terms numerically large while being parametrically small). Unfortunately, if we keep only the lowest order terms in $\mathbf{a}_{S}$, while simultaneously cutting off the perturbation theory integrals at $\Lambda \simeq k_{\mathrm{nl}}$, the theory depends on the cut-off $\Lambda$ unless we work at very small $k$ (where all of the perturbation theory corrections are anyway small). We shall follow the standard practice in the field and take the limit $\Lambda \rightarrow \infty$ when computing the perturbation theory integrals and keep only the lowest order contributions to $\mathbf{a}_{S}$.

\section{Cumulants}

The arguments of section 3 lead us to our expression for the displacement:

$$
\boldsymbol{\Psi}(\mathbf{q})=\boldsymbol{\Psi}_{L}^{(1)}(\mathbf{q})+\mathbf{\Psi}_{L}^{(2)}(\mathbf{q})+\mathbf{\Psi}_{L}^{(3)}(\mathbf{q})+\cdots+\frac{1}{2} \alpha_{1} \nabla \delta_{0}+\mathcal{S}+\cdots
$$

where the first three terms come from the perturbative treatment of the long-wavelength evolution and the last few terms parameterize the impact of the short-wavelength modes on the evolution. The correlation function and power spectrum can now be defined through the cumulants of the displacement. Defining $[6,15]$

$$
K(\mathbf{q}, \mathbf{k})=\left\langle e^{i \mathbf{k} \cdot \boldsymbol{\Delta}}\right\rangle \quad \text { with } \quad \boldsymbol{\Delta}(\mathbf{q})=\mathbf{\Psi}(\mathbf{q})-\mathbf{\Psi}(\mathbf{0})
$$

the power spectrum is

$$
P(k)=\int d^{3} q e^{i \mathbf{q} \cdot \mathbf{k}}[K(\mathbf{q}, \mathbf{k})-1]
$$

\footnotetext{
${ }^{2}$ In terms of $a_{S}$ this term comes from taking $Q_{S}^{i j} \propto \delta^{i j}$ and noting that the long-wavelength modes contribute to $a_{S}$ as tidal fields.
} 
and the correlation function

$$
1+\xi(r)=\int \frac{d^{3} q d^{3} k}{(2 \pi)^{3}} e^{i \mathbf{k} \cdot(\mathbf{q}-\mathbf{r})} K(\mathbf{q}, \mathbf{k})
$$

If only terms quadratic in $k$ are kept in the exponential, the $k$-integral in eq. (4.4) can be done analytically (see e.g. appendix A). The expectation value of the exponential can be obtained using the cumulant theorem $[6,7]$ so we can write

$$
\log K(\mathbf{q}, \mathbf{k})=-\frac{1}{2} k_{i} k_{j} A_{i j}(\mathbf{q})+\frac{i}{6} k_{i} k_{j} k_{\ell} W_{i j \ell}(\mathbf{q})+\cdots
$$

with

$$
\begin{aligned}
A_{i j}(\mathbf{q}) & =2\left\langle\Psi_{i}(\mathbf{0}) \Psi_{j}(\mathbf{0})\right\rangle-2\left\langle\Psi_{i}\left(\mathbf{q}_{1}\right) \Psi_{j}\left(\mathbf{q}_{2}\right)\right\rangle \equiv 2\left(\Sigma^{2} \delta_{i j}-\eta_{i j}\right) \\
W_{i j \ell}(\mathbf{q}) & =\left\langle\Psi_{\{i}\left(\mathbf{q}_{1}\right) \Psi_{j}\left(\mathbf{q}_{2}\right) \Psi_{\ell\}}\left(\mathbf{q}_{2}\right)\right\rangle-\left\langle\Psi_{\{i}\left(\mathbf{q}_{2}\right) \Psi_{j}\left(\mathbf{q}_{1}\right) \Psi_{\ell\}}\left(\mathbf{q}_{1}\right)\right\rangle
\end{aligned}
$$

where we have written $\mathbf{q}=\mathbf{q}_{1}-\mathbf{q}_{2}$ and followed the notation of [15]. Regular LPT can be obtained by Taylor series expanding the exponential and collecting terms in powers of the linear theory power spectrum (which we shall denote as $P_{0}$, for 0-loop). The series expansion so produced agrees with Eulerian perturbation theory [14, 18, 19, 38]. Various useful resummation schemes can be introduced by keeping some of the pieces exponentiated while expanding others (see section 5).

We now consider the contributions to $A_{i j}$ and $W_{i j \ell}$ arising from the various orders in LPT and from the counter terms in eq. (4.1). We shall denote the terms arising from the $1^{\text {st }}$ order in LPT (i.e. Zeldovich approximation) as with a superscript "lin" as we shall want to treat these terms separately on occasion. The other terms we shall denote "lpt" and "eft" depending on their source in eq. (4.1). Thus, e.g.,

$$
A_{i j}(\mathbf{q})=A_{i j}^{\operatorname{lin}}(\mathbf{q})+A_{i j}^{\mathrm{lpt}+\mathrm{eft}}(\mathbf{q})
$$

In the $W_{i j k}$ term, products of two displacement fields appear evaluated at the same point in space, so $W_{i j k}$ is a composite operator which introduces new counter terms. Even though these counter terms can be formally derived from the source, $\mathbf{a}_{S}$, one can also obtain their form based on symmetry arguments (see e.g. ref. [36] for extensive discussion). Considering all two-index quantities which depend at most linearly on $\boldsymbol{\Psi}$ we thus have

$$
\begin{aligned}
\Psi_{i}(\mathbf{q}) \Psi_{j}(\mathbf{q}) & =\Psi_{i}^{(1)}(\mathbf{q}) \Psi_{j}^{(1)}(\mathbf{q})+\Psi_{i}^{(1)}(\mathbf{q}) \Psi_{j}^{(2)}(\mathbf{q})+\Psi_{i}^{(2)}(\mathbf{q}) \Psi_{j}^{(1)}(\mathbf{q})+\cdots \\
& +\frac{1}{3} \alpha_{0} \delta_{i j}+\bar{\alpha}_{2} \delta_{i j} \nabla_{\ell} \Psi_{\ell}^{(1)}+\bar{\alpha}_{3}\left[\nabla_{i} \Psi_{j}^{(1)}+\nabla_{j} \Psi_{i}^{(1)}\right]+\ldots
\end{aligned}
$$

If we were to restrict the perturbation theory integrals to $k<\Lambda$, the coefficients (and their higher-order counterparts) would serve to make the final results $\Lambda$ independent. The $\Lambda$ dependence of these terms is thus set by the structure of the high- $k$ sensitivity in the theory. There will also be a $\Lambda$-independent (or 'finite') piece which can in principle be different for each term.

The terms coming from the small-scale modes, which we shall call the "EFT terms", contribute to $A_{i j}$ as simple integrals of the linear theory power spectrum. For example, the 


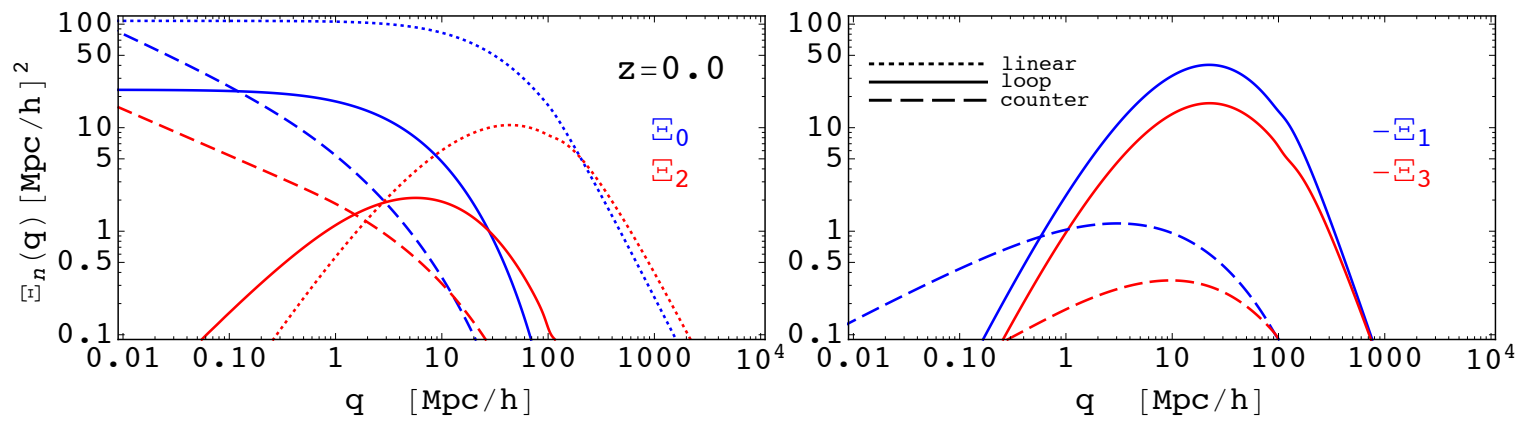

Figure 1. The terms entering into the cumulants of $\boldsymbol{\Psi}$, evaluated at $z=0$, divided into contributions from the linear and 1-loop orders and the counter-terms. The $\Xi_{\ell}$ are defined in section 4 . Note that they are very smooth functions. The linear pieces scale as $D^{2}(z)$ while the 1-loop terms scale as $D^{4}(z)$. The counter terms have been plotted assuming $\alpha_{n}=1$. The $\Xi_{\ell}$ with $\ell>0$ all have a characteristic scale.

cross-term arising from linear theory in $\boldsymbol{\Psi}_{L}$ and the $\nabla \delta$ term gives

$$
\begin{aligned}
\left\langle\Psi_{i}^{(1)}\left(\mathbf{q}_{1}\right) \nabla_{j} \delta\left(\mathbf{q}_{2}\right)\right\rangle & =\left\langle\int \frac{d^{3} p_{1} d^{3} p_{2}}{(2 \pi)^{6}} e^{i\left(\mathbf{p}_{1} \cdot \mathbf{q}_{1}+\mathbf{p}_{2} \cdot \mathbf{q}_{2}\right)} \frac{i \mathbf{p}_{1 i}}{p_{1}^{2}} \delta_{0}\left(\mathbf{p}_{1}\right) i \mathbf{p}_{2 j} \delta_{0}\left(\mathbf{p}_{2}\right)\right\rangle \\
& =\int \frac{d^{3} k}{(2 \pi)^{3}} e^{i \mathbf{k} \cdot \mathbf{q}} P_{0}(k) \frac{k_{i} k_{j}}{k^{2}} \\
& =\frac{1}{3} \xi_{0}(q) \delta_{i j}-\xi_{2}(q)\left(\frac{1}{3} \delta_{i j}-\hat{q}_{i} \hat{q}_{j}\right)
\end{aligned}
$$

with $\xi_{\ell}$ being the usual moments of the linear theory correlation function:

$$
\xi_{\ell}(q) \equiv i^{\ell} \int \frac{k^{2} d k}{2 \pi^{2}} P_{0}(k) j_{\ell}(k q)
$$

The final expressions can be cast in simple form if we introduce $\Xi_{\ell}$ which are extensions of the $\xi_{\ell}$ above. If we write

$$
\begin{aligned}
& \Xi_{0}(q)=\int \frac{d k}{2 \pi^{2}}\left[P_{0}(k)+\frac{9}{98} Q_{1}(k)+\frac{10}{21} R_{1}(k)+\alpha_{1} k^{2} P_{0}(k)\right] j_{0}(k q) \\
& \Xi_{1}(q)=\int \frac{d k}{2 \pi^{2}}\left(-\frac{3}{7 k}\right)\left[Q_{1}(k)-3 Q_{2}(k)+2 R_{1}(k)-6 R_{2}(k)+\alpha_{2} k^{2} P_{0}(k)\right] j_{1}(k q) \\
& \Xi_{2}(q)=\int \frac{d k}{2 \pi^{2}}\left[P_{0}(k)+\frac{9}{98} Q_{1}(k)+\frac{10}{21} R_{1}(k)+\alpha_{1} k^{2} P_{0}(k)\right] j_{2}(k q) \\
& \Xi_{3}(q)=\int \frac{d k}{2 \pi^{2}}\left(-\frac{3}{7 k}\right)\left[Q_{1}(k)+2 Q_{2}(k)+2 R_{1}(k)+4 R_{2}(k)+\alpha_{3} k^{2} P_{0}(k)\right] j_{3}(k q)
\end{aligned}
$$

with $Q_{i}$ and $R_{i}$ the mode-coupling integrals defined in appendix A and $\alpha_{i}$ undetermined constants, then we have

$$
\frac{1}{2} A_{i j}=\frac{1}{3} \delta_{i j}\left(\Xi_{0}(0)+\alpha_{0}-\Xi_{0}(q)\right)+\left(\hat{q}_{i} \hat{q}_{j}-\frac{1}{3} \delta_{i j}\right) \Xi_{2}(q)
$$


where $\alpha_{0}$ is a counter-term with contributions from the zero-lag correlator of $\mathcal{S}$ among other places and we have dropped terms like $\langle\mathcal{S}(\mathbf{q}) \mathcal{S}(\mathbf{0})\rangle$. A similar calculation gives

$$
W_{i j \ell}(q)=\frac{2}{5} \hat{q}_{\{i} \delta_{j \ell\}} \Xi_{1}(q)+\frac{3}{5}\left(5 \hat{q}_{i} \hat{q}_{j} \hat{q}_{\ell}-\hat{q}_{\{i} \delta_{j \ell\}}\right) \Xi_{3}(q)
$$

and the coefficients $\alpha_{2}$ and $\alpha_{3}$ are linear combinations of the $\bar{\alpha}_{2}$ and $\bar{\alpha}_{3}$ we introduced previously. These terms lead to very small corrections and we shall set them to zero henceforth. The common term in $\Xi_{0}$ and $\Xi_{2}$ above is the displacement power spectrum (more details on the derivation of these terms can be found in appendix B of ref. [15] or appendix A of ref. [17]). Note that the $W_{i j \ell}$ term does not have a linear theory contribution and so is expected to be small on large scales. The 'extra' terms, proportional to $\alpha_{1}$, are moments of the linear theory correlation function.

Figure 1 shows these $\Xi_{\ell}$ at $z=0$ as a function of the Lagrangian displacement, $q$. Note that they are all smooth functions and $\Xi_{\ell}$ for $\ell>0$ pick out a characteristic scale. The figure shows the contributions from linear terms, scaling as $D^{2}(z)$, the 1-loop terms, scaling as $D^{4}(z)$, and the EFT terms. If we integrate to finite $k$, or if $P(k)$ has a high- $k$ cut-off, the only non-vanishing contribution as $q \rightarrow 0$ is from $\Xi_{0}$.

At this stage it appears that we have four different counter terms in the 1-loop predictions (eq. 4.14 and $\alpha_{0}$ from eq. 4.16). However, the $\alpha_{0}$ and $\alpha_{1}$ terms are the same, so these two parameters are completely degenerate. The $\alpha_{2}$ and $\alpha_{3}$ terms are not identical, but on large scales $\left(k<0.15 h^{-1} \mathrm{Mpc}\right)$ they are well approximated by the $\alpha_{0}$ term. At smaller scales the differences between these terms becomes significant, but on these scales we may expect 2-loop terms to be large and we leave the regime of validity of our calculation. For these reasons we restrict ourselves henceforth to one free parameter (we choose $\alpha_{0}$ ) and set the other three to zero.

\section{$5 \quad$ Resummation schemes}

Having derived the form for the cumulants we can now use eqs. $(4.3,4.4)$ to compute the 2-point function of the mass, in real space. We show in the next section that expanding the exponentials in a Taylor series reproduces the standard, Eulerian EFT expressions to 1-loop order. By keeping some or all of the terms in the exponential, i.e. (re)summing multiple orders in perturbation theory, we can derive other approximations to the 2-point function. As has been discussed in the literature previously $[15,16,37,38,40]$ one particular advantage of Lagrangian approaches over Eulerian ones is the ability to capture the main effects of advection of mass due to long-wavelength perturbations and to sum the terms in the perturbation theory which scale as the displacement variance times the second derivative of the (linear) correlation function. In $\Lambda \mathrm{CDM}$ models which contain $\mathrm{BAO}$ features even at high order such terms can be numerically quite large, despite being parametrically small.

Unfortunately we are only computing the EFT terms as a series in derivatives, and we need to keep the number of terms small in order to limit the number of free parameters. These terms act to tame (or regularize) the behavior of the perturbation theory terms, so we would like to consistently keep these terms together. Perhaps the simplest way to achieve these goals is to keep in the exponential only $A_{i j}^{\text {lin }}$ while keeping only the $1^{\text {st }}$ order expansion of the other terms. This is also numerically straightforward and efficient. For the power spectrum, for example, we would then have

$$
P(k)=\int d^{3} q e^{i \mathbf{k} \cdot \mathbf{q}-(1 / 2) k_{i} k_{j} A_{i j}^{\mathrm{lin}}}\left[1-\frac{1}{2} k_{i} k_{j} A_{i j}^{\mathrm{lpt}+\mathrm{eft}}+\frac{i}{6} k_{i} k_{j} k_{k} W_{i j k}^{\mathrm{lpt}+\mathrm{eft}}+\cdots\right]
$$




$\begin{array}{ccccc} & z=0.25 & z=0.50 & z=0.75 & z=1.00 \\ \text { LEFT } & -13.6 & -7.8 & -4.8 & -2.7 \\ \text { ZEFT } & -28.5 & -21.9 & -16.2 & -12.75\end{array}$

Table 1. The adopted values of $\alpha_{0}$ for LEFT (eq. 5.1) and ZEFT (eq. 7.4) in figure 2.

and the correlation function can be simplified using the formulae in appendix A as

$$
1+\xi(r)=\int \frac{d^{3} q}{(2 \pi)^{3 / 2}\left|A_{\operatorname{lin}}\right|^{1 / 2}} e^{-(1 / 2)(\mathbf{r}-\mathbf{q})^{T} \mathbf{A}_{\operatorname{lin}}^{-1}(\mathbf{r}-\mathbf{q})}\left[1-\frac{1}{2} G_{i j} A_{i j}^{\mathrm{lpt}+\mathrm{eft}}+\frac{1}{6} \Gamma_{i j k} W_{i j k}^{\mathrm{lpt}+\mathrm{eft}}+\cdots\right]
$$

One of the useful properties of this resummation is that the result on large scales is quite insensitive to the details of the "lpt+eft" terms on small scales, where they are not well constrained. If terms beyond linear are to be kept in the exponent, then care must be taken to ensure the exponentiated matrix is well-behaved and that all of the eigenvalues are positive. After some experimentation we found that the predictions for both $P(k)$ and $\xi(r)$ were relatively insensitive to which terms we kept exponentiated, as long as we consistently treated the zero-lag and $q$-dependent pieces, and we adopted the above procedure due to its numerical and algebraic simplicity. We shall refer to this expression as "CLEFT", since it is an effective field theory in the same spirit as convolution Lagrangian perturbation theory (CLPT; [15]). The integrals can be done, numerically, by simple quadratures. The azimuthal part of the $d^{3} q$ integral gives $2 \pi$ so one needs to do only the integrals over $\mu_{q}$ and $|\mathbf{q}|$. Alternatively one can expand the $\mu$-dependence as in ref. [17] and reduce the remaining integral to a sum of FFTs (see Appendix A for useful formulae).

In the above scheme both the IR-sensitive and UV-sensitive terms in $A_{\text {lin }}$ are kept exponentiated. Other schemes have been put forth. Motivated in part by the poor performance of 'standard' EFT in describing the broadening of the acoustic peak in the correlation function, Senatore and Zaldarriaga put forth an IR-resummation scheme [37] which is similar in spirit to the schemes described above. We describe explicitly the relationship between our schemes and theirs in Appendix B, where we show that their scheme results in resumming a subset of the terms in eq. (5.1). McQuinn \& White [38] put forth a scheme similar to ours except that they resummed only the low- $k$ parts of $A_{\text {lin }}$ while expanding the high- $k$ pieces and the counter terms. They found that the final results were relatively insensitive to the split between the IR- and UV-sensitive pieces, and in fact keeping all of the contributions exponentiated was almost identical to making the split. For this reason we have not attempted such a split here, though it would also be numerically straightforward.

\section{Comparison to Eulerian theory}

It is instructive at this point to illustrate that our prescription regains the standard, Eulerian result. To obtain this we simply expand the exponential in eq. (4.3) and keep terms to order $P_{0}^{2}$. A straightforward calculation (see $[15,17]$ for further details) gives

$$
\begin{aligned}
P(k) & =P_{0}(k)+\frac{9}{98} Q_{1}(k)+\frac{3}{7} Q_{2}(k)+\frac{1}{2} Q_{3}(k)+\frac{10}{21} R_{1}(k)+\frac{6}{7} R_{2}(k)-\alpha_{\text {eft }} k^{2} P_{0}(k) \\
& =P_{0}(k)+P_{1-\text { loop }}^{S P T}-\alpha_{\text {eft }} k^{2} P_{0}(k)
\end{aligned}
$$


where we have written $\alpha_{\text {eft }}=\frac{1}{3} \alpha_{0}-\alpha_{1}-\frac{3}{35}\left(\alpha_{3}-\alpha_{2}\right)$ and indicated the equivalence with the expression for standard, 1-loop perturbation theory in the second line. In the EFT literature this is often written

$$
P(k)=P_{0}(k)+P_{1-\text { loop }}^{S P T}-\left(c_{s}^{2} k_{\mathrm{nl}}^{-2}\right) k^{2} P_{0}(k)
$$

which is obviously of the same form. Note that the coefficient of the $k^{2} P_{0}$ term is a degenerate combination of two different coefficients in the Lagrangian scheme.

\section{Zeldovich EFT}

At this point it is interesting to consider the combination of the lowest order terms in perturbation theory and the lowest order correction from the small-scale terms. Such terms are almost trivial to compute, numerically, and could be expected to capture the essential physics at large scales. The resulting form is also simple enough that generalizations to include redshift-space distortions, bias and reconstruction are relatively straightforward.

In configuration space, keeping only the linear theory LPT terms and the $\nabla \delta$ term all of the required integrals are Gaussians and we have

$$
1+\xi(r)=\int \frac{d^{3} q}{(2 \pi)^{3 / 2}|A|^{1 / 2}} e^{-\frac{1}{2}(\mathbf{r}-\mathbf{q})^{T} \mathbf{A}^{-1}(\mathbf{r}-\mathbf{q})}\left[1-\frac{1}{3} \alpha_{0} \operatorname{tr} G+\cdots\right],
$$

where $\mathbf{A} \equiv \mathbf{A}_{\text {lin }}$ and we have used the notation of [15], reviewed in appendix A. We shall refer to this as the "ZEFT" correlation function, since it is a combination of the Zeldovich approximation and the lowest order EFT terms. The $\operatorname{tr} G$ term scales as a second derivative of $\xi$, and thus this term ${ }^{3}$ modifies the width of the BAO peak at $r \approx 110 h^{-1} \mathrm{Mpc}$. It also leads to a slight increase in the amplitude to smaller $r$.

The expression for the power spectrum is similar

$$
\begin{aligned}
P_{\mathrm{ZEFT}}(k) & =\left(1-\frac{1}{3} \alpha_{0} k^{2}\right) \int d^{3} q e^{i \mathbf{k} \cdot \mathbf{q}} e^{-\frac{1}{2} \mathbf{k}^{T} \mathbf{A k}} \\
& \approx e^{-k^{2} \Sigma^{2}}\left(1-\frac{1}{3} \alpha_{0} k^{2}\right) P_{0}(k) \\
& \approx e^{-k^{2} \Sigma^{2}} P_{0}(k)-\frac{1}{3} \alpha_{0} k^{2} P_{0}(k)
\end{aligned}
$$

where the first term is of the form derived in [13], which can be approximated as an exponential damping of $P_{0}(k)[6,15]$. The width of the damping term, $\Sigma$, is the $1 \mathrm{D} \mathrm{rms}$ displacement computed in linear theory. We give two approximate forms of the power spectrum, which are equal up to terms higher order in $P_{0}$.

\section{Comparison to simulations}

We shall now compare the models described above to the clustering of dark matter measured from a series of N-body simulations. We use 10 simulations run with the TreePM code of [41], each of the same $(\Lambda \mathrm{CDM})$ cosmology but with a different random number seed chosen for the initial conditions. These simulations have been described in more detail elsewhere $[42,43]$, but briefly they were performed in boxes of size $1380 \mathrm{~h}^{-1} \mathrm{Mpc}$ with $2048^{3}$ particles and modeled a $\Lambda \mathrm{CDM}$ cosmology with $\Omega_{m}=0.292, h=0.69, n_{s}=0.965$ and $\sigma_{8}=0.82$. We

\footnotetext{
${ }^{3} \mathrm{~A}$ similar dependence arises to leading-order as the scale-dependent piece of peaks bias, thus this term can do double duty in a theory of biased tracers.
} 

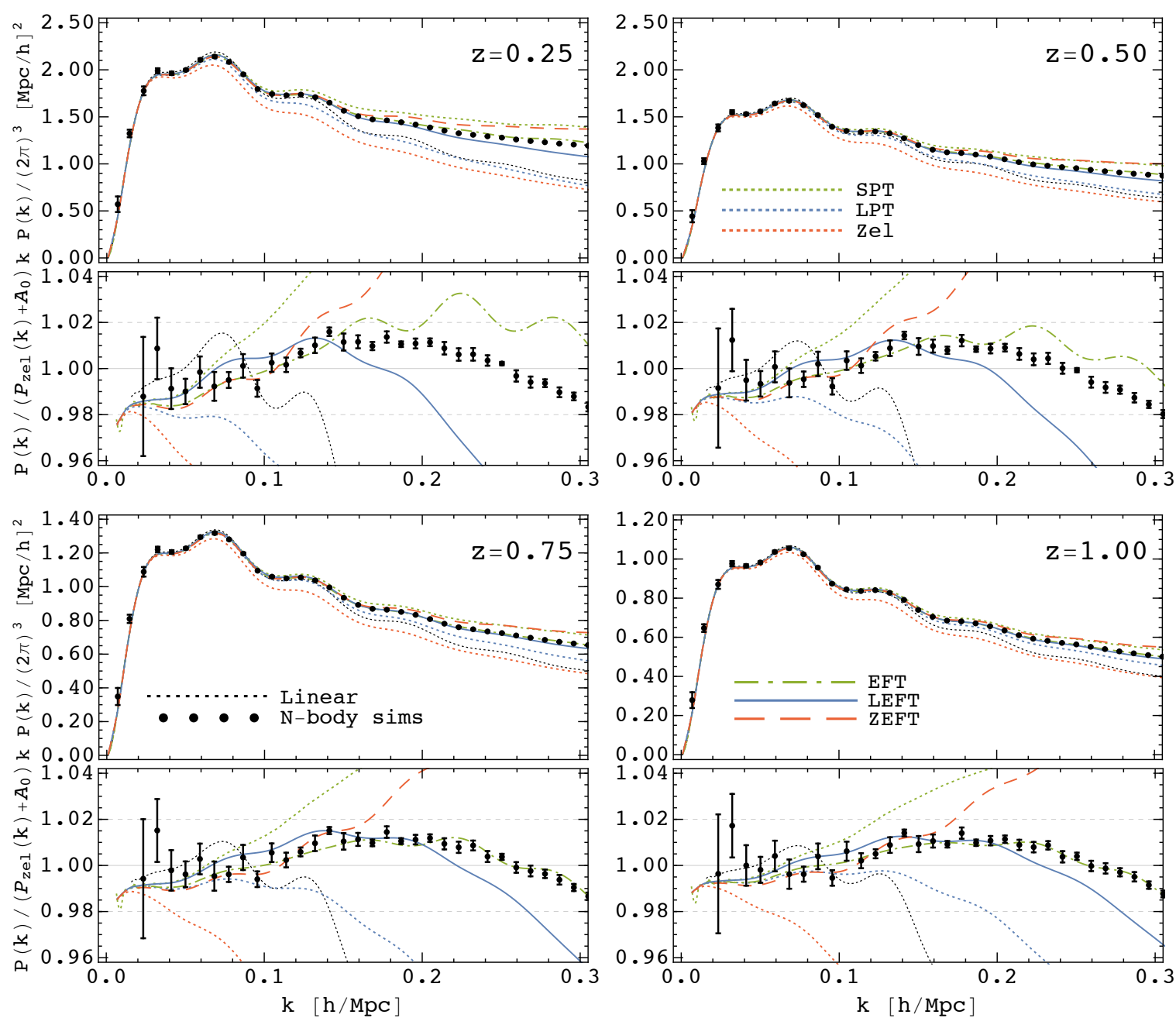

Figure 2. A comparison of the analytic models with N-body simulations at $z=0.25$ (top left), $z=0.5$ (top right), $z=0.75$ (bottom left) and $z=1$ (bottom right). In each panel the black points with error bars represent the mass power spectrum measured from the simulations described in the text. The lines show the different analytical models, described previously and the dotted lines of each color show the perturbation theory results without the EFT terms. The upper panels show $k P(k)$ while the lower panels, with a zoomed-in $y$-axis, show the ratio to a fiducial model (taken to be the Zeldovich power spectrum plus a constant, adjusted as in [29]).

use outputs at $z=0.25,0.50,0.75$ and 1.00 to sample the range of most interest for upcoming large-scale structure surveys. For this cosmology $\Sigma=5.3 h^{-1} \mathrm{Mpc}$ at $z=0.25$, dropping to $4.6 h^{-1} \mathrm{Mpc}$ by $z=0.5,4.1 h^{-1} \mathrm{Mpc}$ by $z=0.75$ and $3.7 h^{-1} \mathrm{Mpc}$ by $z=1$.

Each of the EFT models has at least one free parameter, which we adjust by eye to find the best fit. Since we have this additional freedom we expect the EFT models to fit the N-body data better than the 'standard' perturbation theories. We fix the parameter in Fourier space and use then the same value in configuration space. We compare the clustering with both an absolute scale (chosen to be $k P(k)$ in Fourier space and $r^{2} \xi(r)$ in configuration space) and as a ratio to a fiducial model to enable a better view of the level of agreement. 
We have chosen as our fiducial model a simplified version of the "Zeldovich halo model" of ref. [29]. The fiducial model consists of the Zeldovich power spectrum (section 7) plus a constant, $A_{0}=395,240,145,92 h^{-3} \mathrm{Mpc}^{3}$ at $z=0.25,0.5,0.75$ and 1 . The correlation function of the fiducial model matches the Zeldovich correlation function except at zero lag, which we shall ignore.

Figure 2 compares the Fourier space clustering predicted by the models to that measured in our N-body simulations at various redshifts, i.e. the real-space, mass power spectra. For each redshift the upper panels show the absolute clustering (as $k P(k)$ ) and the lower panels the ratio to our fiducial model. In each panel we have fit the amplitude of the EFT terms by eye to give agreement at the percent level or better at low $k$ while improving the agreement at intermediate scales. Different choices of these parameters can extend or decrease the range of agreement at higher $k$ at the expense of better or worse agreement at intermediate scales, so this comparison depends upon the chosen metric (and thus is subjective). This is particularly true for the Eulerian EFT lines, where much better agreement at high $k$ can be obtained with a slightly worse fit at low $k$. Since our N-body simulations are noisy at low $k$ we have chosen to de-emphasize those points when fitting the EFT model. We note that all of the models fare quite well for scales up to half of the non-linear scale $\left(k_{\mathrm{nl}} \equiv \Sigma^{-1} \sim 0.20 h \mathrm{Mpc}^{-1}\right.$ at $z=0.25,0.22$ at $z=0.5,0.24$ at $z=0.75$ and $k=0.27 \mathrm{~h} \mathrm{Mpc}^{-1}$ at $z=1$ ) and all of the perturbation schemes perform significantly better than linear theory (black dotted line). The Lagrangian EFT appears to match the matter clustering as well as the Eulerian EFT, with the same number of free parameters, at lower $z$ but the Eulerian formulation outperforms the Lagrangian one at higher $z$. Since we know that expanding the exponential in CLEFT would reproduce Eulerian EFT (see section 6) this suggests the exponentiation of $A_{\text {lin }}$ is overdamping the power at high $k$. Eulerian EFT tends to overshoot the N-body points at high $k$ while Lagrangian EFT undershoots. In EFT the $k^{2} P(k)$ term comes in with a negative sign, reducing the well-known overshoot of 1-loop perturbation theory so that it better matches the N-body result (the standard perturbation theory result is shown as the dotted green line). At high $k$ the power goes negative. The addition of the $k^{2} P(k)$ term with a positive coefficient to the Zeldovich power spectrum to get the ZEFT model corrects the well-known short-fall of power in the Zeldovich approximation at high $k$ (the Zeldovich approximation without the $k^{2} P(k)$ term is shown as the dotted line in the lower panels for each redshift). In fact, it appears this addition leads to an overestimate of power at high $k$. The result is very similar whether we use the approximate forms eq. 7.3 or 7.4 and it persists even at high $z$. Such a correction could obviously be tamed by including higher-order terms of the EFT expansion at the cost of introducing further parameters. Figure 2 shows that the 1-loop terms present in the CLEFT but not ZEFT significantly improve the agreement with simulations in the quasi-linear regime because they give more high $k$ power than the Zeldovich approximation and thus require less of the $k^{2} P(k)$ term. At all redshifts the 1-loop CLEFT model agrees with the N-body simulations better than ZEFT, but the agrement is limited to scales sufficiently below $k_{\mathrm{nl}}$. It is interesting that the fiducial model manages to agree with the simulations to nearly as high $k$ as any of the EFTs we have presented, even though it also has only one parameter which could be tuned. We shall return to this comparison later.

Figure 3 makes the same comparison in configuration space, i.e. with the real-space, mass correlation function. Again the boxes show the different redshifts with the upper panels showing the absolute clustering and the lower panels the ratio to our fiducial model (which in this case is simply the Zeldovich approximation). The N-body data are shown as the points, which have significant and highly correlated error bars, while the lines show the same 

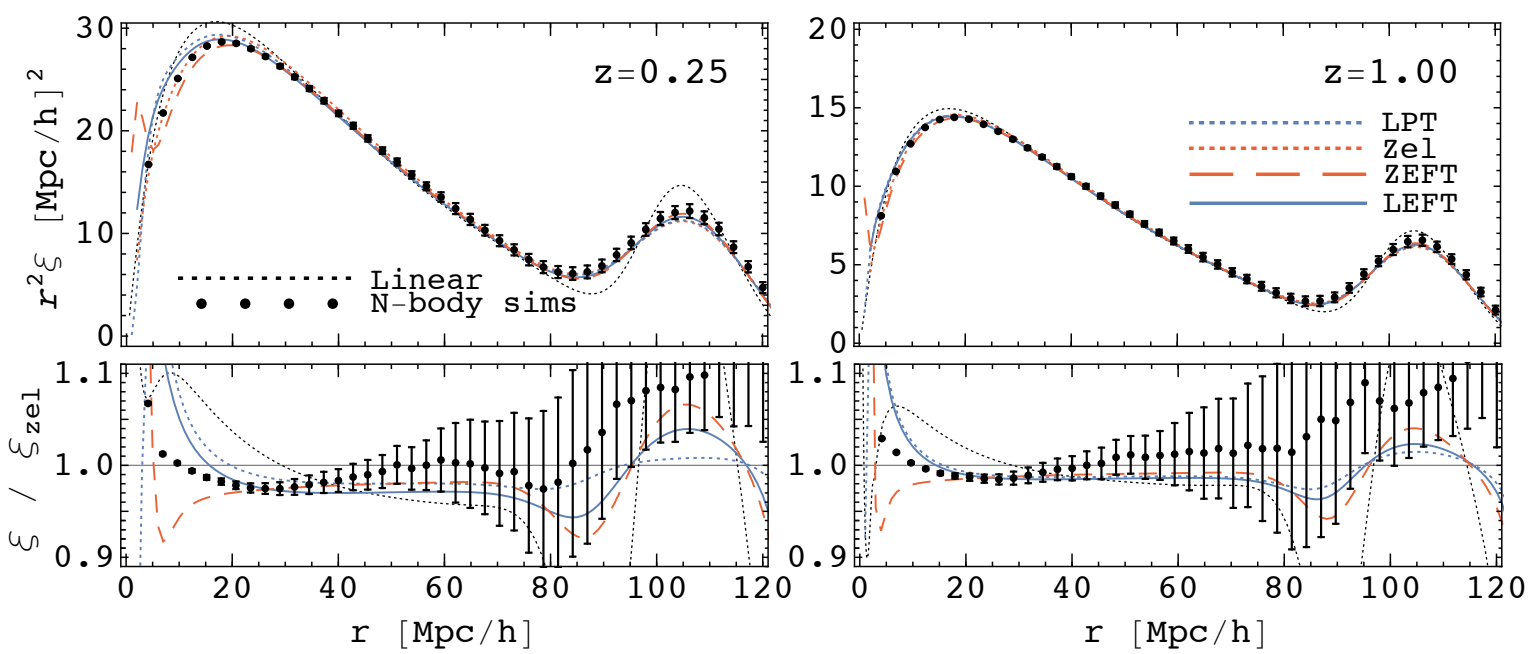

Figure 3. As for figure 2 but in configuration space and with only the $z=0.25$ and $z=1$ results. The upper panels show $r^{2} \xi(r)$ while the lower panels show the ratio to the fiducial model, which is simply the Zeldovich approximation (which is thus not plotted). The error bars, obtained from the scatter between the $10 \mathrm{~N}$-body simulations, are highly correlated.

approximations as above. Here we present only the two end cases, $z=0.25$ and $z=1$, to save space. The Eulerian theories do not make a meaningful prediction for the correlation function, since the power spectrum diverges as $k \rightarrow \infty$. The correlation function in the Lagrangian theories is, however, quite well behaved. As before all of the models fare quite well on scales a factor of $\sim 2-3$ above the non-linear scale, $\Sigma$, and all of the perturbation schemes perform significantly better than linear theory which misses important contributions even at $100 h^{-1} \mathrm{Mpc}$. We note that the Zeldovich approximation does very well on large scales, as has been noted previously $[16,40]$. The CLEFT model is not a significant improvement over ZEFT in configuration space, despite doing much better in Fourier space. The fact that the Zeldovich approximation and ZEFT do so much better in configuration space than in Fourier space indicates that the Fourier space error must transform to small lags in configuration space (see refs. [16, 29] for further discussion).

\section{Conclusions}

Much of the development of perturbation theory in cosmology has focused on an Eulerian description in Fourier space. In this paper we have continued the development of a Lagrangian description, comparing the performance in both Fourier and configuration space. We have extended the traditional perturbative approach using "effective field theory", which amounts to adding to the solution additional terms that are consistent with the symmetries of the problem, are arranged in an expansion in derivatives (powers of $k$ ), and have free coefficients which must be adjusted to fit the data.

Throughout we have tried to make connection with earlier work on this subject, especially refs. $[36,39]$. We provide a slightly different route to the EFT corrections but find the same functional form as these earlier works. Specifically, small-scale physics which is neglected within the usual perturbative calculation gives rise to an additive contribution to 
the displacement field. The lowest order (in derivatives and powers of $\delta_{0}$ ) contribution to $\boldsymbol{\Psi}$ (which transforms as a vector) is proportional to $\nabla \delta_{0}$, with an undetermined coefficient. There could also be an (unconstrained) 'stochastic' term, which is assumed to be small on large scales. If we work at the lowest order, these additional terms modify only the 2-point function of the Lagrangian displacement, adding terms proportional to moments of the linear theory correlation function or closely related functions. These terms serve both to tame the dependence of the perturbation theory on small-scale physics and to improve the agreement with N-body simulations. We show explicitly that 1-loop, Lagrangian EFT is identical to 1-loop, Eulerian EFT if the Lagrangian solution is expanded in powers of the linear theory power spectrum, $P_{0}$. However, the Lagrangian formalism also lends itself to several natural resummation schemes, one of which we adopt here because it is straightforward both algebraically and numerically (section 5). We make explicit comparison with the IR-resummation scheme of ref. [37], showing how to derive their formulae within our framework in appendix B. We choose to resum all of the linear pieces of $A_{i j}$, rather than just the low $k$ terms as in [38]. This leads to little change in the final results.

Comparing the model predictions to the 2-point clustering of the mass in N-body simulations in both Fourier and configuration space, we find that all of the EFT models fare quite well on scales larger than about twice the non-linear scale, but systematically fail as the non-linear scale is approached. All of the perturbative schemes fare better than linear theory on quasi-linear scales. In Fourier space the Lagrangian EFT matches the N-body simulations as well as the Eulerian EFT at low redshifts, but fails at larger scales at high $z$. Since we know that Lagrangian EFT reproduces Eulerian EFT if we expand to a fixed order in the linear power spectrum (section 6) this suggests than our resummation scheme is overdamping power at large $k$. Adding the $k^{2} P(k)$ contribution to the Zeldovich approximation partially corrects the well-known short-fall of power at high $k$ for this model, but leads to an overprediction of power on small scales. Including the 1-loop contributions significantly improves the agreement with the N-body results on quasi-linear scales. The Eulerian EFT does not make a finite prediction for the correlation function, but the Lagrangian form does and the agreement with the simulations is very good on large scales. In fact we find that all of the Lagrangian schemes are in very good agreement with the N-body simulations: including the 1-loop corrections, going from ZEFT to CLEFT, leads to only a modest improvement in the agreement with simulations. The fact that ZEFT does so much better in configuration space than in Fourier space indicates that the Fourier space error must transform to small lags in configuration space, i.e. it must consist primarily of 'broad band' power.

In this paper we have developed and tested a Lagrangian effective field theory to predict the low order clustering of the matter field in real space (addressing many of the same themes as ref. [36]). A C++ code to compute the formulae presented above given a linear theory power spectrum is publicly available at https://github.com/alejandroaviles/CLEFT. One of the advantages of the Lagrangian approach is the relative ease with which redshift-space distortions and bias can be incorporated. We plan to consider these developments in a future paper.

\section{Acknowledgments}

We would like to thank Tobias Baldauf, Matt McQuinn, Uros Seljak and Matias Zaldarriaga for useful discussions and for comments on an early draft of the manuscript. 
Z.V. is supported in part by the U.S. Department of Energy contract to SLAC no. DEAC02-76SF00515. A.A. is supported by the UCMEXUS-CONACyT Postdoctoral Fellowship.

The analysis in this paper made use of the computing resources of the National Energy Research Scientific Computing Center.

\section{A Useful identities}

To simplify the mode coupling integrals we follow [7] and define

$$
R_{n}(k) \equiv \frac{k^{3}}{(2 \pi)^{2}} P_{0}(k) \int_{0}^{\infty} d r P_{0}(k r) \widetilde{R}_{n}(r)
$$

with

$$
\widetilde{R}_{1}=\int_{-1}^{1} d \mu \frac{r^{2}\left(1-\mu^{2}\right)^{2}}{1+r^{2}-2 r \mu} \quad, \quad \widetilde{R}_{2}=\int_{-1}^{1} d \mu \frac{\left(1-\mu^{2}\right) r \mu(1-r \mu)}{1+r^{2}-2 r \mu}
$$

we also use

$$
Q_{n}(k) \equiv \frac{k^{3}}{(2 \pi)^{2}} \int_{0}^{\infty} d r P_{0}(k r) \int_{-1}^{+1} d \mu P_{0}\left(k \sqrt{1+r^{2}-2 r \mu}\right) \widetilde{Q}_{n}(r, \mu)
$$

with

$$
\begin{aligned}
\widetilde{Q}_{1} & =\frac{r^{2}\left(1-\mu^{2}\right)^{2}}{\left(1+r^{2}-2 r \mu\right)^{2}} \\
\widetilde{Q}_{2} & =\frac{\left(1-\mu^{2}\right) r \mu(1-r \mu)}{\left(1+r^{2}-2 r \mu\right)^{2}} \\
\widetilde{Q}_{3} & =\frac{\mu^{2}(1-r \mu)^{2}}{\left(1+r^{2}-2 r \mu\right)^{2}}
\end{aligned}
$$

In the limit of high- $k$ we have $R_{1}(k) \rightarrow(8 / 5)\left(k^{2} \Sigma^{2}\right) P_{0}(k)$ and $R_{2}(k) \rightarrow(-2 / 5)\left(k^{2} \Sigma^{2}\right) P_{0}(k)$.

In performing these calculations one frequently needs to do Gaussian integrals. Again to simplify our notation, and make connection with earlier work, we define

$$
G(\mathbf{k})=e^{-\frac{1}{2} k_{i} k_{j} A_{i j}+i b_{i} k_{i}} \quad, \quad G(\mathbf{b})=\frac{1}{(2 \pi)^{3 / 2}|\mathbf{A}|^{1 / 2}} e^{-\frac{1}{2} b_{i} b_{j} A_{i j}^{-1}}
$$

in terms of which

$$
\begin{aligned}
\int \frac{d^{3} k}{(2 \pi)^{3}} G(\mathbf{k}) & =Q(\mathbf{b}) \\
\int \frac{d^{3} k}{(2 \pi)^{3}} G(\mathbf{k}) k_{a} & =i g_{a} Q(\mathbf{b}) \\
\int \frac{d^{3} k}{(2 \pi)^{3}} G(\mathbf{k}) k_{a} k_{b} & =G_{a b} Q(\mathbf{b}) \\
\int \frac{d^{3} k}{(2 \pi)^{3}} G(\mathbf{k}) k_{a} k_{b} k_{c} & =i \Gamma_{a b c} Q(\mathbf{b})
\end{aligned}
$$

with $\Gamma_{i j k}=\left(\mathbf{A}^{-1}\right)_{i j} g_{k}+\left(\mathbf{A}^{-1}\right)_{k i} g_{j}+\left(\mathbf{A}^{-1}\right)_{j k} g_{i}-g_{i} g_{j} g_{k}, G_{i j}=\left[\left(\mathbf{A}^{-1}\right)_{i j}-g_{i} g_{j}\right]$ and $\mathbf{g}=\mathbf{A}^{-1} \mathbf{b}$ using the notation of Ref. [15]. 
Finally the following integrals are useful when evaluating the power spectrum, as they allow the integral over $d^{3} q$ to be reduced to a sum of 1D integrals:

$$
\begin{aligned}
\int d \mu e^{i \mu A+\mu^{2} B} & =2 e^{B} \sum_{\ell=0}^{\infty}\left(-\frac{2 B}{A}\right)^{\ell} j_{\ell}(A) \\
\int d \mu \mu e^{i \mu A+\mu^{2} B} & =2 i e^{B} \sum_{\ell=0}^{\infty}\left(-\frac{2 B}{A}\right)^{\ell} j_{\ell+1}(A) \\
\int d \mu \mu^{2} e^{i \mu A+\mu^{2} B} & =2 e^{B} \sum_{\ell=0}^{\infty}\left(1+\frac{\ell}{B}\right)\left(-\frac{2 B}{A}\right)^{\ell} j_{\ell}(A) \\
\int d \mu \mu^{3} e^{i \mu A+\mu^{2} B} & =2 i e^{B} \sum_{\ell=0}^{\infty}\left(1+\frac{\ell}{B}\right)\left(-\frac{2 B}{A}\right)^{\ell} j_{\ell+1}(A)
\end{aligned}
$$

\section{B IR resummation}

Senatore and Zaldarriaga have put forth an IR-resummation scheme for EFT [37] which appears different from the schemes we discussed in the main text. In this appendix we show how to derive their scheme from ours. For simplicity we shall restrict ourselves to the Zeldovich approximation, thus $A_{i j}, \Sigma^{2}$ etc. are to be evaluated in linear theory. This already captures all of the main features of the scheme (and indeed most of the main physical effects $[16,40])$ and the generalization to higher orders is straightforward.

We will focus on the power spectrum, which we can write following eq. (4.3) as

$$
\begin{aligned}
P(k) & =\int d^{3} q e^{i \mathbf{k} \cdot \mathbf{q}}\left(e^{-(1 / 2) k_{i} k_{j} A_{i j}}-1\right) \\
& =\int d^{3} q e^{i \mathbf{k} \cdot \mathbf{q}} e^{-k^{2} \Sigma^{2}}\left(e^{k_{i} k_{j} \eta_{i j}}-1\right) \\
& =\int d^{3} q e^{i \mathbf{k} \cdot \mathbf{q}} e^{-k^{2} \Sigma^{2}+k_{i} k_{j} \eta_{i j}}\left(1-e^{-k_{i} k_{j} \eta_{i j}}\right) \\
& =\int d^{3} q e^{i \mathbf{k} \cdot \mathbf{q}} e^{-k^{2} \Sigma^{2}+k_{i} k_{j} \eta_{i j}}\left(k_{i} k_{j} \eta_{i j}-\frac{1}{2}\left[k_{i} k_{j} \eta_{i j}\right]^{2}+\cdots\right) \\
& =\int d^{3} q e^{i \mathbf{k} \cdot \mathbf{q}} e^{-k^{2} \Sigma^{2}+k_{i} k_{j} \eta_{i j}}\left[k_{i} k_{j} \eta_{i j}\right]\left(1+\left[k^{2} \Sigma^{2}-k_{i} k_{j} \eta_{i j}\right]\right) \\
& +\int d^{3} q e^{i \mathbf{k} \cdot \mathbf{q}} e^{-k^{2} \Sigma^{2}+k_{i} k_{j} \eta_{i j}}\left(\frac{1}{2}\left[k_{i} k_{j} \eta_{i j}\right]^{2}-k^{2} \Sigma^{2}\left[k_{i} k_{j} \eta_{i j}\right]\right)+\cdots
\end{aligned}
$$

where we remind the reader that $A_{i j}$ and $\eta_{i j}$ are functions of the Lagrangian displacement, q. If we now define $\mathcal{K}(k, q)=\exp \left[-k^{2} \Sigma^{2}+k_{i} k_{j} \eta_{i j}\right]$ we have

$$
\begin{aligned}
\mathcal{K}^{-1}(k, q) & =\exp \left[k^{2} \Sigma^{2}-k_{i} k_{j} \eta_{i j}\right] \\
\left.\mathcal{K}^{-1}(k, q)\right|_{N} & =\sum_{n=0}^{N} \frac{\left(k^{2} \Sigma^{2}-k_{i} k_{j} \eta_{i j}\right)^{n}}{n !}
\end{aligned}
$$


adopting the notation of [37]. It is useful to note that $k_{i} k_{j} \eta_{i j}$ Fourier transforms to $P_{0}(k)$. Thus

$$
\begin{aligned}
P(k) & =\left.\int d^{3} q e^{i \mathbf{k} \cdot \mathbf{q}} \mathcal{K}(k, q) \mathcal{K}^{-1}(k, q)\right|_{1}\left[k_{i} k_{j} \eta_{i j}\right] \\
& +\int d^{3} q e^{i \mathbf{k} \cdot \mathbf{q}} \mathcal{K}(k, q)\left(\frac{1}{2}\left[k_{i} k_{j} \eta_{i j}\right]^{2}-k^{2} \Sigma^{2}\left[k_{i} k_{j} \eta_{i j}\right]\right)+\cdots
\end{aligned}
$$

If carried through to infinite order this resummation scheme would reproduce the Zeldovich approximation (which our resummation scheme naturally does), but at any finite order some terms are missed.

Our scheme naturally sums all of the linear terms, which has proven to be practically useful to match the broadening of the BAO feature in the correlation function. If we wish to preserve the translation invariance of the theory, there are two obvious ways to do such a resummation. One is to do a 'linearization', as in ref. [37], which includes terms based on their perturbation theory order. The other is the one we have advocated above.

\section{References}

[1] Planck Collaboration, P. A. R. Ade, N. Aghanim, M. Arnaud, M. Ashdown, J. Aumont, C. Baccigalupi, A. J. Banday, R. B. Barreiro, J. G. Bartlett, and et al., Planck 2015 results. XIII. Cosmological parameters, ArXiv e-prints (Feb., 2015) [arXiv:1502.0158].

[2] R. Blandford, the committee for a decadal survey of astronomy and astrophysics 2010, new worlds, New Horizons in Astronomy $\& 3$ Astrophysics, National Research Council, National Academy Press, ISBN-10: 0-309-15799-4 (2014).

[3] Particle Data Group Collaboration, K. Olive et al., Review of Particle Physics, Chin.Phys. C38 (2014) 090001.

[4] F. Bernardeau, S. Colombi, E. Gaztañaga, and R. Scoccimarro, Large-scale structure of the Universe and cosmological perturbation theory, PhysRep 367 (Sept., 2002) 1-248, [astro-ph/0112551].

[5] Y. B. Zel'dovich, Gravitational instability: An approximate theory for large density perturbations., A\&B 5 (Mar., 1970) 84-89.

[6] T. Matsubara, Resumming cosmological perturbations via the Lagrangian picture: One-loop results in real space and in redshift space, PRD $\mathbf{7 7}$ (Mar., 2008) 063530, [arXiv:0711.2521].

[7] T. Matsubara, Nonlinear perturbation theory with halo bias and redshift-space distortions via the Lagrangian picture, PRD $\mathbf{7 8}$ (Oct., 2008) 083519, [arXiv:0807.1733].

[8] T. Buchert, A class of solutions in Newtonian cosmology and the pancake theory, A\&A 223 (Oct., 1989) 9-24.

[9] F. Moutarde, J.-M. Alimi, F. R. Bouchet, R. Pellat, and A. Ramani, Precollapse scale invariance in gravitational instability, ApJ 382 (Dec., 1991) 377-381.

[10] F. R. Bouchet, S. Colombi, E. Hivon, and R. Juszkiewicz, Perturbative Lagrangian approach to gravitational instability., A\&A 296 (Apr., 1995) 575-+, [astro-ph/].

[11] E. Hivon, F. R. Bouchet, S. Colombi, and R. Juszkiewicz, Redshift distortions of clustering: a Lagrangian approach., A\&A 298 (June, 1995) 643, [astro-ph/9407049].

[12] S. Bharadwaj, The Evolution of Correlation Functions in the Zeldovich Approximation and Its Implications for the Validity of Perturbation Theory, ApJ 472 (Nov., 1996) 1-+, [astro-ph/]. 
[13] A. N. Taylor and A. J. S. Hamilton, Non-linear cosmological power spectra in real and redshift space, MNRAS 282 (Oct., 1996) 767-778, [astro-ph/9604020].

[14] C. Rampf and T. Buchert, Lagrangian perturbations and the matter bispectrum I: fourth-order model for non-linear clustering, JCAP 6 (June, 2012) 21, [arXiv:1203.4260].

[15] J. Carlson, B. Reid, and M. White, Convolution Lagrangian perturbation theory for biased tracers, MNRAS 429 (Feb., 2013) 1674-1685, [arXiv: 1209.0780].

[16] M. White, The Zel'dovich approximation, MNRAS 439 (Apr., 2014) 3630-3640, [arXiv: 1401.5466].

[17] Z. Vlah, U. Seljak, and T. Baldauf, Lagrangian perturbation theory at one loop order: Successes, failures, and improvements, PRD 91 (Jan., 2015) 023508, [arXiv:1410.1617].

[18] V. Zheligovsky and U. Frisch, Time-analyticity of Lagrangian particle trajectories in ideal fluid flow, Journal of Fluid Mechanics 749 (June, 2014) 404-430, [arXiv: 1312.6320].

[19] T. Matsubara, Recursive Solutions of Lagrangian Perturbation Theory, ArXiv e-prints (May, 2015) [arXiv: 1505. 0148].

[20] N. Padmanabhan and M. White, Calibrating the baryon oscillation ruler for matter and halos, PRD 80 (Sept., 2009) 063508, [arXiv:0906.1198].

[21] N. McCullagh and A. S. Szalay, Nonlinear Behavior of Baryon Acoustic Oscillations from the Zel'dovich Approximation Using a Non-Fourier Perturbation Approach, ApJ $\mathbf{7 5 2}$ (June, 2012) 21, [arXiv:1202.1306].

[22] S. Tassev and M. Zaldarriaga, The mildly non-linear regime of structure formation, JCAP 4 (Apr., 2012) 13, [arXiv:1109.4939].

[23] N. Padmanabhan, M. White, and J. D. Cohn, Reconstructing baryon oscillations: A Lagrangian theory perspective, PRD 79 (Mar., 2009) 063523-+, [arXiv:0812. 2905].

[24] Y. Noh, M. White, and N. Padmanabhan, Reconstructing baryon oscillations, PRD 80 (Dec., 2009) 123501, [arXiv:0909.1802].

[25] S. Tassev and M. Zaldarriaga, Towards an optimal reconstruction of baryon oscillations, JCAP 10 (Oct., 2012) 6, [arXiv:1203.6066].

[26] M. White, Reconstruction within the Zeldovich approximation, MNRAS 450 (July, 2015) 3822-3828, [arXiv: 1504.0367].

[27] S. Tassev, N-point statistics of large-scale structure in the Zel'dovich approximation, JCAP 6 (June, 2014) 12, [arXiv:1311.6316].

[28] I. Mohammed and U. Seljak, Analytic model for the matter power spectrum, its covariance matrix and baryonic effects, MNRAS 445 (Dec., 2014) 3382-3400, [arXiv:1407.0060].

[29] U. Seljak and Z. Vlah, Halo Zeldovich model and perturbation theory: dark matter power spectrum and correlation function, ArXiv e-prints (Jan., 2015) [arXiv:1501.0751].

[30] D. Baumann, A. Nicolis, L. Senatore, and M. Zaldarriaga, Cosmological non-linearities as an effective fluid, JCAP 7 (July, 2012) 51, [arXiv: 1004.2488].

[31] J. J. M. Carrasco, M. P. Hertzberg, and L. Senatore, The effective field theory of cosmological large scale structures, Journal of High Energy Physics 9 (Sept., 2012) 82, [arXiv:1206.2926].

[32] E. Pajer and M. Zaldarriaga, On the renormalization of the effective field theory of large scale structures, JCAP 8 (Aug., 2013) 37, [arXiv:1301.7182].

[33] A. Manzotti, M. Peloso, M. Pietroni, M. Viel, and F. Villaescusa-Navarro, A coarse grained perturbation theory for the Large Scale Structure, with cosmology and time independence in the UV, JCAP 9 (Sept., 2014) 47, [arXiv:1407.1342]. 
[34] L. Mercolli and E. Pajer, On the velocity in the Effective Field Theory of Large Scale Structures, JCAP 3 (Mar., 2014) 6, [arXiv:1307.3220].

[35] S. M. Carroll, S. Leichenauer, and J. Pollack, Consistent effective theory of long-wavelength cosmological perturbations, PRD 90 (July, 2014) 023518, [arXiv: 1310.2920].

[36] R. A. Porto, L. Senatore, and M. Zaldarriaga, The Lagrangian-space Effective Field Theory of large scale structures, JCAP 5 (May, 2014) 22, [arXiv:1311.2168].

[37] L. Senatore and M. Zaldarriaga, The IR-resummed Effective Field Theory of Large Scale Structures, JCAP 2 (Feb., 2015) 13, [arXiv: 1404.5954].

[38] M. McQuinn and M. White, Cosmological perturbation theory in $1+1$ dimensions, ArXiv e-prints (Feb., 2015) [arXiv: 1502.0738].

[39] T. Baldauf, E. Schaan, and M. Zaldarriaga, On the reach of perturbative descriptions for dark matter displacement fields, ArXiv e-prints (May, 2015) [arXiv: 1505.0709].

[40] S. Tassev, Lagrangian or Eulerian; real or Fourier? Not all approaches to large-scale structure are created equal, JCAP 6 (June, 2014) 8, [arXiv:1311.4884].

[41] M. White, The Mass Function, ApJS 143 (Dec., 2002) 241-255, [astro-ph/0207185].

[42] B. A. Reid, H.-J. Seo, A. Leauthaud, J. L. Tinker, and M. White, A 2.5 per cent measurement of the growth rate from small-scale redshift space clustering of SDSS-III CMASS galaxies, MNRAS 444 (Oct., 2014) 476-502, [arXiv: 1404.3742].

[43] M. White, B. Reid, C.-H. Chuang, J. L. Tinker, C. K. McBride, F. Prada, and L. Samushia, Tests of redshift-space distortions models in configuration space for the analysis of the BOSS final data release, MNRAS 447 (Feb., 2015) 234-245, [arXiv: 1408.5435]. 